\title{
Cardiomiopatia peripartum
}

\author{
Peripartum Cardiomyopathy
}

\author{
Riccardo Raddino, Ivano Bonadei, Melissa Teli, Federica Chieppa, Giorgio Caretta, \\ Debora Robba, Gregoriana Zanini, Enrico Vizzardi, Livio Dei Cas
}

\begin{abstract}
Peripartum Cardiomyopathy. R. Raddino, I. Bonadei, M. Teli, F. Chieppa, G. Caretta, D. Robba, G. Zanini, E. Vizzardi, L. Dei Cas.

Peripartum cardiomyopathy (PPCM) is a rare disorder in which left ventricular dysfunction and symptoms of heart failure occur in the peripartum period in previously healthy women. Incidence of PPCM ranges from 1 in 1300 to 1 in 15,000 pregnancies. The etiology of PPCM is unknown, but viral, autoimmune, and idiopathic causes may contribute. The diagnostic criteria are onset of heart failure in the last month of pregnancy or in the first 5 months postpartum, absence of determinable cause for cardiac failure, and absence of a demonstrable heart disease before the last month of pregnancy. Risk factors for PPCM include advanced maternal age, multiparity, African race, twinning, gestational hy-
\end{abstract}

\begin{abstract}
pertension, and long-term tocolysis. The clinical presentation of patients with PPCM is similar to that of patients with dilated cardiomyopathy. Echocardiography is central to diagnosis. Early diagnosis and initiation of treatment are essential to optimize pregnancy outcome. Treatment is similar to medical therapy for other forms of dilated cardiomyopathy. About half the patients of PPCM recover without complications. The prognosis is poor in patients with persistent cardiomyopathy. Persistence of disease after 6 months indicates irreversible cardiomyopathy and portends worse survival.
\end{abstract}

Keywords: peripartum cardiomyopathy, left ventricular disfunction, heart failure.

Monaldi Arch Chest Dis 2008; 70: 15-23.

\section{Introduzione}

I primi autori che stabilirono una relazione tra scompenso cardiaco e puerperium furono Virchow [1], Ritchie [2] e Porak [3], alla fine del diciannovesimo secolo; tuttavia soltanto nel 1937, dopo che Gouley et al. [4] ebbero osservato gli aspetti clinici e patologici di sette pazienti gravide affette da scompenso cardiaco severo e spesso fatale, tale associazione iniziò ad essere descritta come entità clinica distinta. Le donne presentarono sia negli ultimi mesi di gravidanza sia durante il postpartum una cardiomiopatia dilatativa non ischemica; quattro delle sette pazienti decedettero; l'autopsia mostrò la dilatazione miocardica associata alla presenza di diverse aree necrotiche e fibrotiche molto estese, reperti comunque atipici se paragonati a quelli di altri pazienti con diagnosi di scompenso cardiaco. Per tale motivo gli autori proposero l'esistenza di una relazione, diretta o indiretta, tra scompenso cardiaco, gravidanza e/o puerperium. Analoga associazione fu poi descritta nel $1938 \mathrm{da}$ Hull et al. in 80 pazienti di New Orleans [5], e negli anni successivi diversi autori coniarono i termini di scompenso cardiaco tossico post-partum, malattia cardiaca post-partum, miocardite post-partum, sindrome di Meadow, degenerazione miocardica idiopatica associata a gravidanza, sindrome Zaria e cardiomiopatia post-partum [6-10]. Nel 1971 Demakis et al. [11], in uno studio retrospettivo, descrissero la storia naturale di 27 pazienti con cardiomiopatia associata a gravidanza nel periodo 1947-1967 e definirono tale condizione Cardiomiopatia Peripartum (CMPP), stabilendone i criteri diagnostici, confermati poi durante la Workshop del National Heart, Lung, and Blood Institute (NHLBI) e dell'Office of Rare Diseases of the National Institutes of Health (NIH) nel 2000 [12]: a) scompenso cardiaco insorto nell'ultimo mese di gravidanza o nei 5 mesi dal parto; $b$ ) non disfunzione cardiaca preesistente; c) assenza di cause determinanti cardiomiopatia; più recentemente $d$ ) disfunzione sistolica ventricolare sinistra dimostrata ecocardiograficamente con frazione di eiezione (FE) $<45 \%$ e/o fractional shortening in M-mode $<30 \%$ con dimensione telediastolica $>2.7 \mathrm{~cm} / \mathrm{m}^{2}$ [13-15]. Nonostante i classici criteri per la definizione della CMPP di Demakis et al. limitassero la diagnosi all'ultimo mese gestazionale e ai primi 5 mesi dal parto, diversi articoli pubblicati successivamente descrissero donne con cardiomiopatia più precoce nel corso della gravidanza [16-21]. Infine Elkayam et al., presentando un'ampia casistica di pazienti affette da CMPP, suggerirono il cambiamento dei criteri "classici" dopo aver osservato che sia la presentazione clinica che l'outcome delle pazienti con cardiomiopatia associata alla gravidanza diagnosticata più precocemente rispetto all'ultimo mese di gestazione erano simili a quelle della CMPP "classica", concludendo quindi che entrambe le condizioni potessero rappresentare un continuum della stessa patologia [22]. 


\section{Epidemiologia}

L'incidenza della cardiomiopatia peripartum rimane sconosciuta, rara in alcune parti del mondo, più frequente in altre. Essa varia nei diversi studi considerati ed è stata stimata tra 1:1300 e 1:15000 gestanti $[23,24]$. Questo ampio range probabilmente riflette una sovrastima della patologia nei primi studi, nei quali non venivano ancora considerati $\mathrm{i}$ parametri ecocardiografici diagnostici. Negli Stati Uniti la CMPP è sottodiagnosticata nel 50\% - 75\% dei casi con un'incidenza accettata tra 1000 e 1300 donne per anno [12]. Sempre negli USA è riportata una preferenza geografica per le regioni più meridionali [25]. La tribù Hausa del nord della Nigeria mostra la maggior incidenza nel mondo con 1:100 ed il $13 \%$ circa di tutte le donne [31]; ciò potrebbe essere correlato ad una particolare usanza della tribù consistente nell'ingestione di un particolare sale di lago durante l'immediato periodo post-partum, pratica che indurrebbe un aumento significativo del postcarico [32-33].

\section{Fattori di rischio}

I fattori di rischio includono età $>30$ anni, obesità, preeclampsia ed ipertensione arteriosa, abuso di alcol, tabacco e cocaina, anamnesi familiare positiva e bassa situazione socio-economica $[4,11,23$, $25,34-37]$. Non è ancora chiaro il ruolo della razza come fattore di rischio indipendente oppure sia dovuto all'interazione con l'ipertensione; infatti Elkayam [22] ha dimostrato che negli Stati Uniti la CMPP non è limitata alle donne di colore poiché questo studio non supporta un'associazione tra multiparità e sviluppo della patologia in quanto quasi il $40 \%$ dei casi si è verificato nella prima gravidanza e più del $50 \%$ con la seconda. Diversi lavori hanno anche considerato il ruolo della malnutrizione, della mancanza di cure prenatali e l'allattamento al seno [4], nonostante alcuni studi successivi non ne avessero dimostrato l'evidenza [8,38]. Anche la localizzazione geografica può contribuire: alcuni lavori indicano un'aumentata incidenza nelle regioni tropicali con maggiore temperatura ed umidità $[4,36]$. Una terapia tocolitica prolungata con beta-agonisti è risultata associata con lo sviluppo di CMPP in quanto tali agenti potrebbero smascherare la cardiomiopatia sottostante [39]. Tuttavia finché i fattori di rischio non potranno essere delineati con precisione, è difficile porre raccomandazioni per lo screening della popolazione più a rischio.

\section{Eziologia}

L'eziologia di questa patologia rimane incerta, tuttavia è stata proposta una gamma di possibili cause includenti la miocardite, la risposta autoimmune alla gravidanza, un male adattamento agli stress emodinamici della gestazione inclusa l'attivazione di citochine, infezione virale sistemica e tocolisi prolungata. Inoltre sono stati riportati diversi lavori sulla CMPP familiare [42-44], evidenziando la possibilità che alcuni casi di tale patologia potessero es- sere collegati ad una CMD familiare smascherata dalla gravidanza. Sfortunatamente, con l'eccezione di alcuni case reports, non esistono in letteratura significativi dati patologici post-mortem utili per definire meglio l'eziologia dei pazienti con CMPP. Comunque ci sono più evidenze che supportano la miocardite o un processo autoimmune come causa di malattia rispetto alle altre eziologia proposte. Le possibili ipotesi sono discusse in seguito.

\section{a. Miocardite}

La relazione tra gravidanza e miocardite virale fu evidenziata per la prima volta nel 1968 da Farber e Glasgow [45]. I loro studi su animali dimostrarono che le femmine gravide sono più suscettibili alle infezioni virali rispetto a quelle non gravide, in quanto notarono che i virus si moltiplicavano maggiormente nei cuori delle ultime. La gravidanza potrebbe predisporre le donne ad una forma più severa di miocardite, soprattutto in caso di infezioni da virus cardiotropi [38]. I cambiamenti fisiologici ed emodinamici della gestazione possono risultare in una aumentata suscettibilità alla miocardite virale, maggiore carica virale (come Coxsackie ed Echovirus) e peggioramento delle lesioni miocardiche $[45,46]$. Alcuni studi immunologici sulle donne hanno dimostrato aumentata attività di linfociti T suppressor durante la gestazione [47], che potrebbero aumentare la suscettibilità alle infezioni virali $[48,49]$.

Biopsie miocardiche in donne con CMPP hanno poi dimostrato la presenza di miocardite in molte delle pazienti, anche se in percentuale marcatamente differente nei vari studi $(8.8-76 \%)[19,38,50-53]$. Tuttavia alcuni di questi lavori non hanno raggiunto la significatività statistica a causa della scarsa grandezza dei campioni. La maggior incidenza di miocardite in CMPP (76\%) è stata riportata da Midiei et al. [51]; questi autori eseguirono biopsie endomiocardiche su pazienti con sintomi di scompenso cardiaco congestizio al momento della presentazione ed inclusero, in tale percentuale, sia quelli con miocardite istologica borderline sia coloro con miocardite attiva.

In seguito Rizeq et al. [19], eseguirono uno studio retrospettivo su biopsia e CMPP, rivelando una bassa incidenza di miocardite $(8.8 \%)$; tale incidenza fu comparabile ad una popolazione di controllo suddivisa per sesso ed età sottoposta a trapiando cardiaco per CMD idiopatica $(9.1 \%)$. Il motivo di questa discrepanza tutt'ora non è chiaro; alcuni suggeriscono che il momento della biopsia in relazione all'inizio della sintomatologia possa essere un fattore significativo [19, 51], altri fattori includono le difficoltà nello stabilire la diagnosi di miocardite con la biopsia endomiocardica $[54,55]$, la variabilità nell'inclusione di pazienti con miocardite borderline e quelli con miocardite istologica definita dai criteri di Dallas e la potenziale variabilità geografica delle popolazioni di pazienti affette.

\section{b. Meccanismi autoimmuni}

Molti studi hanno suggerito un possibile ruolo dell'autoimmunità associata alla gravidanza nell'e- 
ziologia della CMPP; questa infatti si potrebbe sviluppare solo in uno specifico contesto di fattori immuni e genetici alterati [59-63]. In letteratura sono stati riportati elevati titoli anticorpali contro proteine selettive per il tessuto cardiaco e livelli significativamente aumentati di Tumor Necrosis Factor$\alpha$, interleuchina- 6 e recettori solubili di Fas [56, 57], a loro volta correlati alle dimensioni del ventricolo sinistro e a una più bassa frazione di eiezione $[57,58]$.

Warrach et al. [64] valutarono la concentrazione di Immunoglobuline (classe $\mathrm{G}$ e relative sottoclassi G1, G2, G3) contro la miosina cardiaca in 47 pazienti affette da cardiomiopatia peripartum di diverse regioni del mondo, rispetto ad una popolazione di controllo di madri sane e pazienti affette da MCD idiopatica; rispetto ai controlli con MCD, nei quali si evidenziava una up-regulation selettiva delle immunoglobuline $\mathrm{G}$, nella CMPP risultavano elevati i valori di tutte le sottoclassi di tali anticorpi. Inoltre tutte le pazienti con IgG3 positive si trovavano in una classe NYHA peggiore, suggerendo un possibile utilizzo di queste immunoglobuline nella valutazione prognostica della CMPP. D'altro canto era già stato dimostrato in precedenza che aumentati livelli di IgG3 predicevano una prognosi peggiore a 6 mesi nonostante terapia medica ottimizzata in pazienti affetti da scompenso cardiaco cronico [65].

Alcuni autori hanno ipotizzato che dopo il parto la degenerazione rapida dell'utero si esplica nella frammentazione del tropocollagene da parte di enzimi collageno litici che rilasciano actina, miosina e loro metaboliti [66]; si formano così anticorpi anti actina che a loro volta cross-reagiscono con il miocardio determinando quindi lo sviluppo di una cardiomiopatia. Tale teoria, tuttavia, non spiega il motivo per cui la CMPP si presenta prima del parto.

\section{c. Infezione virale}

Nei campioni bioptici endomiocardici di pazienti affette da cardiomiopatia peripartum è stata riscontrata una componente infiammatoria, verosimilmente dovuta a riattivazione di un'infezione virale latente come conseguenza ad immunodepressione durante la gravidanza [101], tuttavia non esistono attualmente risultati significativi riguardo la presenza di un genoma virale in miocardiociti di donne con CMPP.

È stato recentemente pubblicato uno studio condotto su 26 pazienti affette da CMPP con riscontro di genoma virale (Parvovirus B 19, Herpes virus umano, Epstein Barr virus e Citomegalovirus) in 8 donne $(30,7 \%)$, associato a infiammazione interstiziale [102]. Questo risultato suggerisce un'elevata prevalenza di modificazioni infiammatorie a causa virale in una non bassa percentuale di pazienti con cardiomiopatia peripartum.

In uno studio effettuato da Bachmaier et al. [103] sono stati valutati alcuni dati che supportano l'ipotesi sul ruolo della Chlamydia nella patogenesi della CMPP. Infatti la presenza di un peptide della catena pesante dell' $\alpha$-miosina muscolo specifica del cuore di topo, la quale ha una sequenza omologa alla proteina di membrana appartenente ai tre ge- neri principali di Chlamydia, è causa di una miocardiopatia autoimmune-infiammatoria nei topi; 1'iniezione di peptidi omologhi di Chlamydia nel ratto induce infiammazione vascolare, fibrosi ed occlusione di vasi cardiaci. Tali osservazioni supportano l'origine infettiva della patologia. Il ruolo della Chlamydia è stato poi ulteriormente osservato in uno studio condotto in Nigeria [104]: il 96\% delle pazienti affette da CMPP erano positive per IgG anti Chlamydia, contro 1'80\% dei controlli, all'analisi sierologica.

È stato inoltre osservato che il riscontro di un elevato titolo anticorpale, sia del tipo G che A, contro Chlamydia e pneumococco ha un ruolo negativo dal punto di vista prognostico [105]. Altri studi retrospettivi suggeriscono una relazione tra CMPP e malattie sessualmente trasmissibili [106].

\section{d. Altre eziologie}

Sia la scarsa nutrizione che le carenze alimentari, specialmente di selenio e tiamina, sono state associate a cardiomiopatia peripartum [25, 107-110], nonostante studi successivi avessero smentito tali associazioni $[111,112]$. In letteratura è stata segnalata anche l'associazione CMPP - strenuo esercizio aerobico durante l'ultimo trimestre di gravidanza [113]. È stato inoltre proposto il ruolo dell'edema polmonare acuto in corso di crisi ipertensiva [114, 115], sebbene l'ipertensione, di solito transitoria e di grado lieve, possa essere assente. È stato già riportato in precedenza il ruolo dell'assunzione di sale nelle donne Nigeriane.

\section{Presentazione clinica e diagnosi}

Dai criteri già enunciati nell'introduzione si può evincere che la diagnosi di CMPP dovrebbe essere presa in considerazione allorquando una donna gravida presenti scompenso cardiaco, nonostante si debba tenere presente che molte donne sane presentano nell'ultimo mese di gravidanza normale dispnea, edemi declivi ed astenia.

Sintomi e segni che devono far sospettare scompenso cardiaco comprendono dispnea parossistica notturna, dolore toracico, tosse notturna, comparsa di nuovi soffi cardiaci, elevata pressione venosa giugulare ed epatomegalia.

Nell'introduzione a questa revisione sono già stati descritti i criteri clinici ed ecocardiografici per la diagnosi di CMPP; in modo particolare questi ultimi permettono una diagnosi più accurata escludendo i cosidetti "falsi positivi" della sola valutazione clinica, errore molto frequente in autori nell'"era pre-ecocardiografica". Nei loro lavori, infatti, essi hanno incluso pazienti affette da ipertensione non controllata, disfunzione diastolica, infezione sistemica, embolia polmonare o complicanze proprie della gravidanza avanzata (es. preeclampsia). Per tale motivo è stato proposto, come criterio addizionale per la diagnosi, la presenza di un nuovo peggioramento della funzione ventricolare sinistra all'ecocardiogramma [12-14, 40].

L'elettrocardiogramma è solitamente caratterizzato da ritmo sinusale o tachicardia sinusale, ma in 
rari casi potrebbe evidenziare la presenza di un'aritmia. Altre anomalie, seppur raramente presenti, sono ipertrofia ventricolare sinistra, onde $\mathrm{T}$ invertite, onda Q e alterazioni aspecifiche del tratto ST [117].

Il ruolo della biopsia endomiocardica è ancora controverso. La sensibilità diagnostica di tale procedura pare essere del $50 \%$ mentre la specificità molto elevata (99\%); è stata osservata un'elevata percentuale di falsi negativi.

Diagnosi differenziale deve essere fatta con infarto miocardico acuto, sepsi, severa pre-eclampsia, embolia del liquido amniotico ed embolia polmonare; per la diagnosi di cardiomiopatia peripartum bisogna avere anzitutto un forte sospetto associato alla presenza contemporanea di sintomi, in rapporto alla gravidanza, ed all'identificazione ecocardiografica di una disfunzione ventricolare sinistra di recente insorgenza.

\section{Complicanze}

La presenza di trombi endocavitari è spesso osservata nelle pazienti con grave disfunzione sistolica $(\mathrm{FE}<35 \%)$ associata ad un'elevata incidenza di mortalità dovuta a trombo embolia. L'embolia sistemica può portare ad attacchi ischemici transitori, emiplegia, embolia polmonare, infarto acuto del miocardio, occlusione acuta dell'arteria mesenterica superiore che si manifesta sottoforma di addome acuto, infarto renale e splenico. È stata inoltre osservata nelle pazienti con CMPP la presenza di tachicardia sinusale, tachiaritmie atriali e ventricolari, battiti ectopici ventricolari e persino la sindrome di Wolfe-Parkinson-White. Inoltre è stata evidenziata una maggior incidenza di aborti e parti prematuri. In alcuni casi sono state descritte anomalie fetali congenite $(4-6 \%)$.

\section{Trattamento}

In genere il trattamento della cardiomiopatia peripartum è simile a quello dello scompenso cardiaco congestizio $[12,118]$ in gravidanza e quindi nella riduzione dell'introito di sale con la dieta e nell'utilizzo di diuretici per ridurre la congestione polmonare ed il sovraccarico ventricolare [4, 117]. L'utilizzo di ACE-inibitori è controindicato per il forte rischio teratogeno, per l'insufficienza renale neonatale e per il rischio di morte neonatale [119]; tuttavia nel post-parto, anche nelle donne che allattano, tali farmaci rappresentano il caposaldo della terapia.

L'idralazina è il farmaco d'elezione in gravidanza, aggiunta ai nitrati e/o amlodipina; ciononostante alcuni calcio antagonisti, a causa del loro effetto inotropo negativo, dovrebbero essere evitati [120].

Per quanto riguarda i betabloccanti è stato dimostrato che il carvedilolo migliora la sopravvivenza nelle donne gravide affette da CMD ma è ancora sconosciuto il suo ruolo nella CMPP [121]. L'uso dei beta bloccanti a lungo termine, in gravidanza, pare essere associato a un basso peso alla nascita nel bambino [122].

Nel trattamento delle pazienti altamente sintomatiche i farmaci che riducono sia il precarico che il postcarico (nitroprussiato e nitroglicerina) e quelli inotropi (dobutamina, dopamina, milrinone) potrebbero essere presi in considerazione; bisogna però porre particolare attenzione al nitroprussiato poiche il suo principale metabolita, il tiocianato, si può accumulare nel feto [123].

In uno studio randomizzato [51] è stato suggerito che l'immunosoppressione potrebbe essere utile nelle donne con miocardite associata a cardiomiopatia peripartum. Tuttavia sarebbe meglio non usare tale terapia in quanto non è ancora conosciuto il suo ruolo modulatore nella risposta immune [124].

L'uso di immunoglobuline e.v. sembra migliorare la funzione ventricolare sistolica sinistra nelle pazienti affette da CMD e miocardite [138-139].

Alcuni studi hanno evidenziato un'elevata incidenza di tromboembolia in questa popolazione di donne, comprese embolie coronariche [125], trombi biventricolari [126-128], embolie polmonari [129] e periferiche [130] ed infarti cerebrali su base trombotica [131]. Tutto ciò porrebbe indicazione all'utilizzo di eparina a basso peso molecolare (5000 UI 2 volte/die) mentre l'uso di dicumarolici dovrebbe essere evitato per il loro già dimostrato effetto teratogeno [132].

Un ruolo a parte è rivestito dal trapianto cardiaco; già nel 1987 Avarot et al. pubblicarono i loro primi risultati riguardo a sei casi di CMPP sottoposti a trapianto cardiaco [133], riportando due morti postoperatorie attribuibili a rigetto. Un altro gruppo di ricercatori [112] descrissero che due delle loro cinque pazienti affette da CMPP sopravvissero per sette mesi dopo il trapianto ed una di loro fu sottoposta ad uno nuovo a causa del rigetto. Il gruppo di Keogh [134], analizzando il decorso post-trapianto in 10 pazienti, concluse che le donne affette da CMPP avevano un'incidenza più elevata di rigetto nei primi 6 mesi ed una maggiore incidenza di infezioni gravi, così come altri autori riscontrarono una mortalità del $25 \%$ nello stesso periodo di tempo.

\section{Prognosi}

La prognosi della CMPP dipende dall'entità del recupero della frazione d'eiezione. Infatti le pazienti con severa disfunzione sistolica ventricolare sinistra hanno minori possibilità di sopravvivere e recuperare una normale funzionalità cardiaca. Un valore della frazione di accorciamento inferiore al $20 \%$ ed un diametro telediastolico superiore a $60 \mathrm{~mm}$ al momento della diagnosi predice un rischio relativo superiore a 3 per la disfunzione ventricolare sinistra persistente [14].

Vi sono studi in cui circa il $50 \%$ delle pazienti affette da CMPP recuperano la funzione ventricolare di base a 6 mesi circa dalla diagnosi $[11,140]$ mentre ne esistono altri che hanno mostrato che pazienti con severa disfunzione cardiaca hanno poca possibilità di recuperare tale funzione [15].

Un indice prognostico positivo è correlabile anche alla riserva contrattile inotropa, correlata ad una ripresa della funzione ventricolare sinistra durante eco-stress dobutamina [141, 142]. 


\section{Outcome gravidico}

Nelle donne affette da una persistente disfunzione cardiaca la gravidanza è sconsigliata, per il rischio di peggioramento progressivo della funzionalità ventricolare sinistra. Circa il 20\% delle donne affette da CMPP sopravvivono soltanto per essere state sottoposte a trapianto cardiaco e la maggior parte di queste recuperano solo parzialmente la funzione ventricolare sinistra.

Il gruppo di Elkayam [143], ha dimostrato che, nelle donne con CMPP anamnestica, una successiva gravidanza potrebbe essere associata ad un pessimo outcome materno e fetale sia nelle donne con funzionalità persistentemente depressa, sia in quelle che recuperano tale funzione dopo un peggioramento iniziale $[11,139]$.

Sulla base delle raccomandazioni della Task Force dell'ESC nel 2003 [144] sarebbe indicato l'utilizzo dell'ecocardiografia in pazienti con cardiomiopatia dilatativa (CMD) certa o sospetta e/o anamnesi familiare positiva per CMD, sconsigliata un'eventuale gravidanza nelle donne con FE depressa e, nelle donne gravide affette da CMD, in caso di peggioramento della sintomatologia, il ricovero urgente.

\section{Conclusioni e prospettive future}

La cardiomiopatia peripartum rappresenta una rara ma severa forma di insufficienza cardiaca ad eziologia ignota ad insorgenza durante l'ultimo periodo di gravidanza oppure durante l'inizio del puerperium. La diagnosi di questa affezione include l'evidenza ecocardiografica di disfunzione ventricolare sinistra che rientra nei criteri diagnostici modificati; alla luce di questi ultimi dovrebbero essere rivisti $\mathrm{i}$ fattori di rischio e quelli eziologici.

Tuttavia, sebbene siano stati fatti molti sforzi per approfondire la conoscenza nei confronti della CMPP, dovrebbero essere ancora raggiunti degli obiettivi sia per migliorare la qualità di vita che per diminuire la mortalità delle pazienti affette da questa patologia.

La creazione di un registro nazionale potrebbe rivestire un ruolo fondamentale per aumentare la comprensione della cardiomiopatia peripartum; ciò potrebbe comportare una migliore valutazione dei fattori di rischio, della storia clinica individuale, della diagnosi e dell'efficacia del trattamento mediante analisi epidemiologica. Il corretto utilizzo di questi registri potrebbe portare ad amplificare le raccomandazioni della già citata Task Force dell'ESC fino ad individuare delle linee guida a disposizione dell'intera comunità medica.

A proposito delle linee di ricerca future, dovrà essere chiarito il ruolo dei processi autoimmuni, dell'apoptosi e del ruolo dei virus nella patogenesi della CMPP. Una miglior comprensione della fisiopatologia potrà condurre a nuove strategie terapeutiche in considerazione anche dell'evidenza sempre più concreta della morte cellulare programmata dei miociti, come responsabile della progressione dello scompenso cardiaco; in quest'ottica una terapia antiapoptotica rappresenterebbe una futura prospettiva per lo sviluppo di terapie che prevengono sia l'instaurarsi che la progressione della cardiomiopatia peripartum.

\section{Riassunto}

La cardiomiopatia peripartum (CMPP) è una rara malattia in cui la disfunzione ventricolare sinistra e i sintomi di scompenso cardiaco compaiono nel periodo peripartum in donne precedentemente sane. L'incidenza della CMPP è compresa tra $1 \mathrm{su}$ 1300 e 1 su 15000 partorienti. L'eziologia della CMPP è sconosciuta, ma sono state identificate cause virali, autoimmuni e idiopatiche. I criteri diagnostici sono la comparsa di scompenso cardiaco nell' ultimo mese di gravidanza e nei primi cinque mesi post-partum, assenza di cause che possano determinare scompenso cardiaco e assenza di una dimostrabile patologia cardiaca prima dell'ultimo mese di gravidanza. I fattori di rischio per la CMPP includono avanzata età materna, multiparità, razza africana, gemellarità, ipertensione gravidica. La diagnosi precoce e l' inizio del trattamento sono essenziali per ottimizzare l'esito della gravidanza. Il trattamento è una terapia medica simile a quella per le altre forme di cardiomiopatia dilatativa. Circa la metà delle pazienti con CMPP si riprende senza complicazioni. La persistenza della patologia dopo sei mesi indica una cardiomiopatia irreversibile e preannuncia una peggior sopravvivenza.

Parole chiave: cardiomiopatia peripartum, disfunzione ventricolare sinistra, scompenso cardiaco.

\section{Bibliografia}

1. Virchow, R. Sitzing der Berliner Geburtshilflisher Gersellskhalt, cited by Porak, C. De l'influence réciproque de la grossesse et des maladies du Coeur, thesis, Paris, 1880.

2. Ritchie C. Clinical contribution to the patho-diagnosis and treatment of certain chronic diseases of the heart. $E d$ inburgh Med J 1850; 2: 2.

3. Porak, C. De l'influence réciproque de la grossesse et des maladies du Coeur, thesis, Paris, 1880.

4. Gouley BA, McMillan TM, bellet S. Idiopathic myocardial degeneration associated with pregnancy and especially the puerperium. Am J Med Sci 1937; 19: 185-99.

5. Hull E, Hidden E. Postpartal heart failure. South Med J 1938; 31: 265-70.

6. Veille JC. Peripartum cardiomyopathies: a review. Am J Obstet Gynecol 1984; 148: 805-18.

7. Sakakibara S, Sekiguchi M, Konno S, Kusumoto M. Idiopathic postpartum cardiomyopathy: report of a case with special reference to its ultrastructural changes in the myocardium as studies by endomyocardial biopsy. Am Heart J 1970; 80: 385-95.

8. Manolio TA, Baughman KL, Rodeheffer R, et al. Prevalence and etiology of idiopathic cardiomyopathy (summary of a National Heart, Lung, and Blood InstituteWorkshop). Am J Cardiol 1992; 69: 1458-66.

9. Julian DG, Szekely P. Peripartum cardiomyopathy. Prog Cardiovasc Dis 1985; 27: 223-40.

10. Danbauchi SS. Echocardiographic features of peripartum cardiac failure: the Zaria syndrome. Trop Doct 2002; 32: 24-7.

11. Demakis JG, Rahimtoola SH, Sutton GC, et al. Natural course of peripartum cardiomyopathy. Circulation 1971; 44: 1053-61. 
12. Pearson GD, Veille JC, Rahimtoola S, et al. Peripartum cardiomyopathy. National Heart Lung and Blood Institute and Office of Rare Diseases (National Institutes of Health) workshop recommendations and review. JAMA 2000; 283: 1183-8.

13. Hibbard JU, Lindheimer M, Lang RM. A modified definition for peripartum cardiomyopathy and prognosis based on echocardiography. Obstet Gynecol 1999; 94: 311-6.

14. Chapa JB, Heiberger HB, Weinert L, Decara J, Lang RM, Hibbard JU. Prognostic value of echocardiography in peripartum cardiomyopathy. Obstet Gynecol 2005; 105: 1303-8.

15. Witlin AG, Mabie WC, Sibai BM. Peripartum cardiomyopathy: a longitudinal echocardiographic study. $\mathrm{Am} \mathrm{JOb-}$ stet Gynecol 1997; 177: 1129-32.

16. Brown G, O'Leary M, Douglas I, Herkes R. Perioperative management of a case of severe peripartum cardiomyopathy. Anaesth Intensive Care 1992; 20: 80-3.

17. Forssell G, Laska J, Olofsson C, Olsson M, Mogensen L. Peripartum cardiomyopathy: three cases. J Intern Med 1994; 235: 493-6.

18. Yahagi N, Kumon K, Nakatani T. Peripartum cardiomyopathy and tachycardia followed by multiple organ failure. Anesth Analg 1994; 79: 581-2.

19. Rizeq MN, Rickenbacher PR, Fowler MB, Billingham ME. Incidence of myocarditis in peripartumcardiomyopathy. Am J Cardiol 1994; 74: 474-7.

20. Mellor DJ, Bodenham A. Infiltration anesthesia in the management of caesarian section in a patient with peripartum cardiomyopathy. Med Trop 1996; 56: 376-80.

21. Alvarez Navascues R, Marin R, Testa A, Paneda F, Alvarez Grande J. Preeclampsia and peripartum cardiomyopathy: infrequent association. Nefrologia 2001; 21: 84-7.

22. Elkayam U, Akhter MW, Singh H, et al. Pregnancy-associated cardiomyopathy: clinical characteristics and a comparison between early and late presentation. Circulation 2005; 111: 2050-5.

23. Cunningham FG, Pritchard JA, Hankins GD, Anderson PL, Lucas MJ, Armstrong KF. Peripartum heart failure: Idiopathic cardiomyopathy or compounding cardiovascular events. Obstet Gynecol 1986; 67: 157-68.

24. Cetta F, michels VV. The natural history and spectrum of idiopathic dilated cardiomyopathy, including HIV and peripartum cardiomyopathy. Curr Opin Cardiol 1995; 10: $332-8$

25. Homans DC. Peripartum cardiomyopathy. $N$ Engl J Med 1985; 312: 1432.

26. Fett JD, Carraway RD, Dowell DL, King ME, Pierre R. Peripartum cardiomyopathy in the Hospital Albert Schweitzer District of Haiti. Am J Obstet Gynecol 2002; 186: 1005-10.

27. Fett JD, Christie LG, Carraway RD, Sundstrom JB, Ansari AA. Learning from a population-based peripartum cardiomyopathy registry. Circulation 2004; 110 (III): 727.

28. Fett JD, Dowell DL, Carraway RD, Sundstrom JB, Ansari AA. One hundred cases of peripartum cardiomyopathy... and counting: what is going on? Int J Cardiol 2004; 97: 571-3.

29. Ferriere M, Sacrez A, Bouhour JB, et al. La myocardiopathie du peripartum: aspects actuels. Etude multicentrique: 11 observations. Arch Mal Coeur 1990; 83: 1563-9.

30. Cénac A, Djibo A. Postpartum cardiac failure in Sudanese-Sahelian Africa: clinical prevalence in western Niger. Am J Trop Med Hyg 1998; 58: 319-23.

31. Davidson NM, Parry EH. Peri-partum cardiac failure. $Q J$ Med 1978; 47: 431-61.

32. Sanderson JE, Adesanya CO, Anjorin FI, Parry EH. Postpartum cardiac failure-heart failure due to volume overload? Am Heart J 1979; 97: 613.

33. Fillmore SJ, Parry EH. The evolution of peripartal heart failure in Zaria. Circulation 1977; 56: 1058.
34. Lang RM, Lampert MB, Poppas A, et al. In: Elkayam U, Gleicher N, editors. Cardiac problem in pregnancy: diagnosis and management of maternal and fetal disease. 3rd Ed. New York: Wiley-Liss; 1998. p. 87.

35. Witlin AG, Mabie WC, Sibai BM. Peripartum cardiomyopathy: an ominous diagnosis. Am J Obstet Gynecol 1997; 176: $182-8$.

36. Desai D, Moodley J, Naidoo D. Peripartum cardiomyopathy: experiences at King Edward VIII Hospital, Durban, South Africa and a review of the literature. Trop Doct 1995; 25: 118-23.

37. Heider AL, Kuller JA, Strauss RA, Wells SR. Peripartum cardiomyopathy: a review of the literature. Obstet Gynecol Surv 1999; 54: 526-531.

38. O'Connell JB, Costanzo-Nordin MR, Subramanian R, et al. Peripartum cardiomyopathy: clinical, hemodynamic, histologic, and prognostic characteristics. J Am Coll Cardiol 1986; 8: 52-6.

39. Lampert MB, Hibbard J, Weinert L, Briller J, Lindheimer M, Lang RM. Peripartum heart failure associated with prolonged tocolytic therapy. Am J Obstet Gynecol 1993; 168: 493-5.

40. Lampert MB, Lang RM. Peripartum cardiomyopathy. Am Heart J 1995; 130: 860-70.

41. van Hoeven KH, Kitsis RN, Katz SD, Factor SM. Peripartum versus idiopathic dilated cardiomyopathy in young women - a comparison of clinical pathologic, and prognostic features. Int J Cardiol 1993; 40: 57-65.

42. Pierce JA, Price BO, Joyce JW. Familial occurrence of postpartal heart failure. Arch Intern Med 1963; 111: 651-5.

43. Pearl W. Familial occurrence of peripartum cardiomyopathy. Am Heart J 1995; 129: 421-2.

44. Massad LS, Reiss CK, Mutch DG, Hasket EJ. Family peripartum cardiomyopathy after molar pregnancy. $\mathrm{Ob}-$ stet Gynecol 1993; 81: 886-8.

45. Farber PA, Glasgow LA. Viral myocarditis during pregnancy: encephalomyocarditisvirus infection in mice. $\mathrm{Am}$ Heart J 1970; 80: 96-102.

46. Lyden DC, Huber SA. Aggravation of coxsackie virus, group B, type- 3-induced myocarditis and increase in cellular immunity to myocyte antigens in pregnant Balb/c mice and animal treated with progesterone. Cell Immunol 1984; 87: 462-72.

47. Kovithavongs T, Dossetor JB. Suppressor cells in human pregnancy. Transplant Proc 1978; 10: 911-3.

48. Thong YH, Steele RW, Vincent MM, Hensen SA, Bellanti JA. Impaired in vitro cell-mediated immunity to rubella virus during pregnancy. N Engl J Med 1973; 289: 604-6.

49. D'Cruz IA, Balani SG, Iyer LS. Infectious hepatitis and pregnancy. Obstet Gynecol 1968; 31: 449-55.

50. Sanderson JE, Olsen EG, Gatei D. Peripartum heart disease: an endomyocardial biopsy study. Br Heart J 1986; 56: 285-91.

51. Midei MG, DeMent SH, Feldman AM, Hutchins GM, Baughman KL. Peripartum myocarditis and cardiomyopathy. Circulation 1990; 81: 922-8.

52. Melvin KR, Richardson PJ, Olsen EG, Daly K, Jackson G. Peripartum cardiomyopathy due to myocarditis. $N$ Engl J Med 1982; 307: 731-4.

53. Felker GM, Jaeger CJ, Klodas E, et al. Myocarditis and long-term survival in peripartum cardiomyopathy. Am Heart J 2000; 140: 785-91.

54. Chow LH, Radio SH, Sears TD, McManus BM. Insensitivity of right ventricular endomyocardial biopsy in the diagnosis of myocarditis. J Am Coll Cardiol 1989; 14: 915-20.

55. Hauck AJ, Kearney DL, Edwards WD. Evaluation of post-mortem endomyocardial biopsy specimens from 38 patients with lymphocytic myocarditis: implications for the role of sampling error. Mayo Clin Proc 1989; 64: $1235-45$. 
56. Ansari AA, Neckelmann N, Wang YC, Gravanis MB, Sell KW, Herskowitz A. Immunologic dialogue between cardiac myocytes, endothelial cells and mononuclear cells. Clin Immunol Immunopathol 1993; 68: 208-14.

57. Sliwa K, Skudicky D, Bergemann A, Candy G, Puren A, Sareli P. Peripartum cardiomyopathy: analysis of clinical outcome, left ventricular function, plasma levels of cytokines and Fas/APO-1. J Am Coll Cardiol 2000; 35: 701-5.

58. Sliwa, K., Forster, O., Libhaber, E., Fett, J.D., Sundstrom, J.B., Hilfiker-Kleiner, D., Ansari, A.A. Peripartum cardiomyopathy: inflammatory markers as predictors of outcome in 100 prospectively studied patients. Eur Heart $J$ 2005 Sep 5; [Epub ahead of print].

59. Klintschar M, Schwaiger P, Mannweiler S, Regauer S, Kleiber M. Evidence of fetal microchimerism in Hashimoto's thyroiditis. J Clin Endocrinol Metab 2001; 86: 2494-8.

60. Miyashita Y, Ono M, Ono M, Ueki H, Kurasawa K. Y chromosome microchimerism in rheumatic autoimmune disease. Ann Rheum Dis 2000; 59: 655-6.

61. Johnson KL, Nelson JL, Furst DE. Fetal cell microchimerism in tissue from multiple sites in women with systemic sclerosis. Arthritis Rheum 2001; 44: 1848-54.

62. Ansari AA, Fett JD, Carraway RE, Mayne AE, Onlamoon N, Sundstrom JB. Autoimmune mechanisms as the basis for human peripartum cardiomyopathy. Clin Rev Allergy Immunol 2002; 23: 301-24.

63. Sundstrom JB, Fett JD, Carraway RD, Ansari AA. Is peripartum cardiomyopathy an organ-specific autoimmune disease? Autoimmun Rev 2002; 1: 73-7.

64. Warraich RS, Sliwa K, Damasceno A, et al. Impact of pregnancyrelated heart failure on humoral immunity: clinical relevance of G3- subclass immunoglobulins in peripartum cardiomyopathy. Am Heart $J$ 2005; 150: 263-9.

65. Warraich RS, Young JB, Sestier F, et al. Clinical and prognostic relevance of Ig-G3 reactivity in heart failure: a substudy of vasogen's immune modulation therapy in patients with chronic heart failure. J Am Coll Cardiol 2004; 43: 226A.

66. Knobel B, Melamud E, Kishon Y. Peripartum cardiomyopathy. Isr J Med Sci 1984; 20: 1061-3.

67. Liu Y, Cigola E, ChengW, et al. Myocyte nuclear mitotic division and programmed myocyte cell death characterize the cardiac myopathy induced by rapid ventricular pacing in dogs. Lab Invest 1995; 73: 771-87.

68. Sharov VG, Sabbah HN, Shimoyama H, Goussev AV, Lesch M, Goldstein S. Evidence of cardiocyte apoptosis in myocardium of dogs with chronic heart failure. $A m J$ Pathol 1996; 148: 141-9.

69. Li Z, Bing OH, Long X, Robinson KG, Lakatta EG. Increased cardiomyocyte apoptosis during the transition to heart failure in the spontaneously hypertensive rat. Am $J$ Physiol 1997; 272: H2313-9.

70. Adams JW, Sakata Y, Davis MG, et al. Enhanced Goq signaling: a common pathway mediates cardiac hypertrophy and apoptotic heart failure. Proc Natl Acad Sci U S A 1998; 95: 10140-5.

71. Hirota H, Chen J, Betz UA, et al. Loss of a gp130 cardiac muscle cell survival pathway is a critical event in the onset of heart failure during biomechanical stress. Cell 1999; 97: 189-98.

72. Condorelli G, Morisco C, Stassi G, et al. Increased cardiomyocyte apoptosis and changes in proapoptotic and antiapoptotic genes bax and bcl-2 during left ventricular adaptations to chronic pressure overload in the rat. Circulation 1999; 99: 3071-8.

73. Geng YJ, Ishikawa Y, Vatner DE, et al. Apoptosis of cardiac myocytes in Gs transgenic mice. Circ Res 1999; 84: 34-42.

74. Zhang D, Gaussin V, Taffet GE, et al. TAK1 is activated in the myocardium after pressure overload and is suffi- cient to provoke heart failure in transgenic mice. Nat Med 2000; 6: 556-63.

75. Xing H, Zhang S, Weinheimer C, Kovacs A, Muslin AJ. 14-3-3 proteins block apoptosis and differentially regulate MAPK cascades. EMBO J 2000; 19: 349-58.

76. Bisognano JD, Weinberger HD, Bohlmeyer TJ, et al. Myocardialdirected overexpression of the human beta(1)adrenergic receptor in transgenic mice. $J \mathrm{Mol} \mathrm{Cell} \mathrm{Cardi-}$ ol 2000; 32: 817-30.

77. Sam F, Sawyer DB, Chang DL, et al. Progressive left ventricular remodeling and apoptosis late after myocardial infarction in mouse heart. Am J Physiol 2000; 279: H422-8.

78. Palojoki E, Saraste A, Eriksson A, et al. Cardiomyocyte apoptosis and ventricular remodeling after myocardial infarction in rats. Am J Physiol 2001; 280: H2726-31.

79. Narula J, Haider N, Virmani R, et al. Apoptosis in myocytes in endstage heart failure. N Engl J Med 1996; 335: 1182-9.

80. Olivetti G, Abbi R, Quaini F, et al. Apoptosis in the failing human heart. $N$ Engl J Med 1997; 336: 1131-41.

81. Saraste A, Pulkki K, Kallajoki M, et al. Cardiomyocyte apoptosis and progression of heart failure to transplantation. Eur J Clin Invest 1999; 29: 380-6.

82. Guerra S, Leri A, Wang X, et al. Myocyte death in the failing human heart is gender dependent. Circ Res 1999; 85: 856-66.

83. Haider N, Narula N, Narula J. Apoptosis in heart failure represents programmed cell survival, not death, of cardiomyocytes and likelihood of reverse remodeling. $J$ Card Fail 2002; 8: 512-7.

84. Nadal-Ginard B, Kajstura J, Leri A, Anversa P. Myocyte death, growth, and regeneration in cardiac hypertrophy and failure. Circ Res 2003; 92: 139-50.

85. Dorn II GW, Brown JH. Gq signaling in cardiac adaptation and maladaptation. Trends Cardiovasc Med 1999; 9: 26-34.

86. D'Angelo DD, Sakata Y, Lorenz JN, et al. Transgenic Goq overexpression induces cardiac contractile failure in mice. Proc Natl Acad Sci U S A 1997; 94: 8121-6.

87. Sakata Y, Hoit BD, Liggett SB, Walsh RA, Dorn II GW. Decompensation of pressure-overload hypertrophy in Gqoverexpressing mice. Circulation 1998; 97: 1488-95.

88. Thornberry NA, Lazebnik Y. Caspases: enemies within. Science 1998; 281: 1312-6.

89. Ashkenazi A, Dixit VM. Death receptors: signaling and modulation. Science 1998; 281: 1305-8.

90. Green DR, Reed JC. Mitochondria and apoptosis. Science 1998; 281: 1309-12.

91. Jeremias I, Kupatt C, Martin-Villalba A, et al. Involvement of CD95/ Apo1/Fas in cell death after myocardial ischemia. Circulation 2000; 102: 915-20.

92. Lee P, Sata M, Lefer DJ, Factor SM,Walsh K, Kitsis RN. Fas pathway is a critical mediator of cardiac myocyte death and MI during ischemia-reperfusion in vivo. Am J Physiol Heart Circ Physiol 2003; 284: H456-63.

93. Bialik S, Cryns VL, Drincic A, et al. The mitochondrial apoptotic pathway is activated by serum and glucose deprivation in cardiac myocytes. Circ Res 1999; 85: 403-14.

94. Malhotra R, Brosius III FC. Glucose uptake and glycolysis reduce hypoxia-induced apoptosis in cultured neonatal rat cardiac myocytes. J Biol Chem 1999; 274: 12567-75.

95. von Harsdorf R, Li PF, Dietz R. Signaling pathways in reactive oxygen species-induced cardiomyocyte apoptosis. Circulation 1999; 99: 2934-41.

96. de Moissac D, Gurevich RM, Zheng H, Singal PK, Kirshenbaum LA. Caspase activation and mitochondrial cytochrome $\mathrm{c}$ release during hypoxia-mediated apoptosis of adult ventricular myocytes. J Mol Cell Cardiol 2000; 32: 53-63.

97. Kang PM, Haunstetter A, Aoki H, Usheva A, Izumo S. Morphological and molecular characterization of adult 
cardiomyocyte apoptosis during hypoxia and reoxygenation. Circ Res 2000; 87: 118-25.

98. Adams JW, Pagel AL, Means CK, Oksenberg D, Armstrong RC, Brown JH. Cardiomyocyte apoptosis induced by $\mathrm{Gq}$ signaling is mediated by permeability transition pore formation and activation of the mitochondrial death pathway. Circ Res 2000; 87: 1180-7.

99. Yussman MG, Toyokawa T, Odley A, et al. Mitochondrial death protein Nix is induced in cardiac hypertrophy and triggers apoptotic cardiomyopathy. Nat Med 2002; 8: 725-30.

100. Hayakawa Y, Chandra M, Miao W, et al. Inhibition of cardiac myocyte apoptosis improves cardiac function and abolishes mortality in the peripartum cardiomyopathy of Goq transgenic mice. Circulation 2003; 108: 3036-41.

101. Murphy JG. Peripartum cardiomyopathy. In: Cooper LT, editor. Myocarditis: from bench to bedside. Totowa (NJ): Humana Press; 2003. p. 589-608.

102. Bultmann BD, Klingel K, Nabauer M, Wallwiener D, Kandolf R. High prevalence of viral genomes and inflammation in peripartum cardiomyopathy. Am J Obstet Gynecol 2005; 193: 363-5.

103. Bachmaier K, Neu N, de la Maza LM, Pal S, Hessel A, Penninger JM. Chlamydia infections and heart disease linked through antigenic mimicry. Science 1999; 283: 1335-9.

104. Cenac A, Djibo A, Sueur JM, Chaigneau C, Orfila J. Chlamydia infection and peripartum dilated cardiomyopathy in Niger. Med Trop (Mars) 2000; 60: 137-40.

105. Cenac A, Djibo A, Chaigneau C, Velmans N, Orfila J. Are anti- Chlamydia pneumoniae antibodies prognosis indicators for peripartum cardiomyopathy? J Cardiovasc Risk 2003; 10: 195-9.

106. Arnould N, Diemunsch P, Raiga J, Brettes JP. Peripartum dilated cardiomyopathies: is there a correlation with sexually transmitted diseases? Gynecol Obstet Fertil 2002; 30: 59-63.

107. Walsh JJ, Burch GE, Black WC, Ferrans VJ, Hibbs RG. Idiopathic myocardiopathy of the puerperium (postpartal heart disease). Circulation 1965; 32: 19-31.

108. Seftel H, Susser M. Maternity and myocardial failure in African women. Br Heart J 1961; 23: 43-52.

109. Kothari SS. Aetiopathogenesis of peripartum cardiomyopathy: prolactin-selenium interaction? Int J Cardiol 1977; 60: 111.

110. Blegen SD. Post-partum congestive heart failure. Beriberi heart disease. Acta Med Scand 1965; 178: 515-24.

111. Fett JD, Ansari AA, Sundstrom JB, Combs GF. Peripartum cardiomyopathy: a selenium disconnection and an autoimmune connection. Int J Cardiol 2002; 86: 311-6.

112. Costanzo-Nordin MR, O'Connell JB. Peripartum cardiomyopathy in the 1990's etiologic and prognostic considerations and review of the literature. In: Zipes DP, Rowlands DJ, editors. Progress in cardiology. Philadelphia, PA: Lea and Febiger; 1989. p. 225-39.

113. Stamler J, Horowitz SF, Goldman ME, Matza D, Machac J. Peripartum cardiomyopathy: a role for cardiac stress determinants other than pregnancy? Mt Sinai J Med 1989; 56: 285-9.

114. Brockington IF. Postpartum hypertensive heart failure. Am J Cardiol 1971; 27: 650-8.

115. Phillips Jr JH, Burch GE. A review of cardiovascular diseases in the White and Negro races. Medicine 1960; 39: 241-88 (Baltimore).

116. Adesanya CO. Peripartum heart failure - a reappraisal of the concept. In: Akinkugbe OO, editor. Cardiovascular disease in Africa. Ikega, Nigeria: Ciba-Geigy; 1976. p. 325 .

117. Brown CS, Bertolet BD. Peripartum cardiomyopathy: a comprehensive review. Am J Obstet Gynecol 1998; 178: 409-14.

118. Rahimtoola SH, Tak T. The use of digitalis in heart failure. Curr Probl Cardiol 1996; 21: 781-853.
119. Mastrobattisha JM. Angiotensin converting enzyme inhibitors in pregnancy. Semin Perinatol 1997; 21: 124-34.

120. Packer M, O'Connor CM, Ghali JK, et al. Effect of amlodipine on morbidity and mortality in severe chronic heart failure. Prospective Randomized Amlodipine Survival Evaluation Study Group. N Engl J Med 1996; 335: 1107-14.

121. Packer M, Bristow MR, Cohn JN, et al. The effect of carvedilol on morbidity and mortality in patients with chronic heart failure. N Engl J Med 1996; 334: 1349-55.

122. Lip AYH, Beevers M, Churchill D, Shaffer LM, Beevers DG. Effect of atenolol on birth weight. Am J Cardiol 1997; 79: 1436-8.

123. Stempel JE, O'Grady JP, Morton MJ, Johnson KA. Use of sodium nitroprusside in complications of gestational hypertension. Obstet Gynecol 1982; 60: 533-8.

124. Mason JW, O'Connell JB, Herskowitz A, et al. A clinical trial of immunosuppressive therapy for myocarditis. The Myocarditis Treatment Trial Investigators. $N$ Engl J Med 1995; 333: 269-75.

125. Box LC, Hanak V, Arciniegas JG. Dual coronary emboli in peripartum cardiomyopathy. Tex Heart Inst $J$ 2004; 31 : 442-4.

126. Nishi I, Ishimitsu T, Ishizu T, et al. Peripartum cardiomyopathy and biventricular thrombi. Circ J 2002; 66: 863-5.

127. Damorou FJ, Kane A, Napporn G, et al. Biventricular thrombus complicating peripartum cardiomyopathy. A case report. Dakar Med 2000; 45: 199-201.

128. Sanchez-Rubio Lezcano J, Galache Osuna JG, Marquina Barcos A, Calvo Cebollero I, Diarte de Miguel JA, Placer Peralta LJ. Peripartum cardiomyopathy with biventricular thrombi. Ann Med Intern 2004; 21: 498-500.

129. Quinn B, Doyle B, McInerney J. Postnatal pre-cordial pain. Pulmonary embolism or peripartum cardiomyopathy. Emerg Med J 2004; 21: 746-7.

130. Bennani SL, Loubaris M, Lahlou I. Postpartum cardiomyopathy revealed by acute lower limb ischemia. Ann Cardiol Angeiol 2003; 52: 382-5 (Paris).

131. Kaufman I, Bondy R, Benjamin A. Peripartum cardiomyopathy and thromboembolism; anesthetic management and clinical course of an obese, diabetic patient. Can J Anaesth 2003; 50: 161-5.

132. Pettifor JM, Benson R. Congenital malformations associated with the administration of oral anticoagulants during pregnancy. J Pediatr 1975; 86: 459-62.

133. Aravot DJ, Banner NR, Dhalla N, et al. Heart transplantation for peripartum cardiomyopathy. Lancet 1987; 2: 1024.

134. Keogh A, Macdonald P, Spratt P, Marshman D, Larbalestier R, Kaan A. Outcome in peripartum cardiomyopathy after heart transplantation. $J$ Heart Lung Transplant 1994; 13: 202-7.

135. Rickenbacher PR, Rizeq MN, Hunt SA, Billingham ME, Fowler MB. Long-term outcome after heart transplantation for peripartum cardiomyopathy. Am Heart J 1994; 127: 1318-23.

136. Kereiakes DJ, Parmley WW. Myocarditis and cardiomyopathy. Am Heart J 1984; 108: 1318-26.

137. Dec Jr GW, Palacios IF, Fallon JT, et al. Active myocarditis in the spectrum of acute dilated cardiomyopathies. Clinical features, histologic correlates, and clinical outcome. N Engl J Med 1985; 312: 885-90.

138. Drucker NA, Colan SD, Lewis AB, et al. Gamma-globulin treatment of acute myocarditis in the pediatric population. Circulation 1994; 89: 252-7.

139. Bozkurt B, Villaneuva FS, Holubkov R, et al. Intravenous immune globulin in the therapy of peripartum cardiomyopathy. J Am Coll Cardiol 1999; 34: 177-80.

140. Sutton MS, Cole P, Plappert M, Saltzman D, Goldhaber S. Effects of subsequent pregnancy on left ventricular function in peripartum cardiomyopathy. Am Heart $J$ 1991; 121: 1776-8.

141. Dorbala S, Brozena S, Zeb S, et al. Risk stratification of women with peripartum cardiomyopathy at initial presen- 


\section{CARDIOMIOPATIA PERIPARTUM}

tation: a dobutamine stress echocardiography study. $J$ Am Soc Echocardiogr 2005; 18: 45-8.

142. Lampert MB, Weinert L, Hibbard J, Korcarz C, Lindheimer M, Lang RM. Contractile reserve in patients with peripartum cardiomyopathy and recovered left ventricular function. Am J Obstet Gynecol 1997; 176: 189-95.

143. Elkayam U, Tummala PP, Rao K, et al. Maternal and fetal outcomes of subsequent pregnancies in women with peripartum cardiomyopathy. N Engl J Med 2001; 344: 1567-71.

144. Expert consensus document on management of cardiovascular diseases during pregnancy.The Task Force on the Management of Cardiovascular Diseases During Pregnancy of the European Society of Cardiology. European Heart Journal 2003; 24: 761-781. 